\title{
|Instalación y configuración de NFS en CentOS 7
}

\author{
Installation and configuration of NFS in CentOS 7
}

\author{
Ignacio Hernandez-Martinez ${ }^{a}$, Yareli Hernandez-Zurita ${ }^{b}$, Laura A. Zapata-Hernandez ${ }^{b}$, Luis
}

A. Alarcon-Arenas ${ }^{b}$, Leonel Velazquez-Hernandez ${ }^{b}$, Raul H. Palacios ${ }^{c}$

\begin{abstract}
:
Currently, there are protocols for the transfer or distribution of files that allow remote access to a specific document located within a specific directory of the server and make some modification or simply reading this document. This practice refers to the NFS protocol, compatible by default with UNIX operating systems and mostly LINUX distributions. It is a very popular protocol for distributed file systems and allows remote access to the server's directory by its client users.
\end{abstract}

Keywords:

Protocols, Remote Access, NFS Server, NFS, NFS Client.

\section{Resumen:}

Actualmente, existen protocolos para la transferencia o distribución de archivos que permiten acceder de manera remota a un determinado documento ubicado dentro de un directorio específico del servidor y realizar alguna modificación o simplemente la lectura de este documento. Esta práctica hace referencia al protocolo NFS, compatible por defecto con sistemas operativos UNIX y en su mayoría distribuciones LINUX. Es un protocolo muy popular para sistemas de archivos distribuidos y permite acceso remoto al directorio del servidor por parte de sus usuarios cliente.

Palabras Clave:

Protocolos, Acceso remoto, Servidor NFS, NFS, Cliente NFS.

\section{Introducción}

\section{CentOS 7}

La distribución de CentOS Linux es una plataforma estable, predecible, manejable y reproducible derivada de las fuentes de Red Hat Enterprise Linux (RHEL) [1].

NFS (Network File System) es un popular protocolo de sistema de archivos distribuidos en una red local de computadoras que permite a los usuarios compartir directorios remotamente a través de un servidor de archivos y conectarse a este para compartir información entre los usuarios de la red [2] [3].
NFS permite aprovechar el espacio de almacenamiento en una ubicación diferente y también permite escribir en el mismo espacio desde múltiples servidores o clientes sin esfuerzo. Es bastante útil para directorios a los que los usuarios necesitan acceder con frecuencia [4] [5].

En la literatura se han encontrado diferentes implementaciones del sistema NFS enfocado a usos diversos [6] [7].

\section{Cliente}

Cualquier elemento de un sistema de información que requiere un servicio mediante el envío de solicitudes al servidor. Cuando dos programas se comunican por una

\footnotetext{
Autor de correspondencia, Universidad Autónoma del Estado de Hidalgo, Escuela Superior de Huejutla, Email: naxito15.martinez@gmail.com
}

b Universidad Autónoma del Estado de Hidalgo, Escuela Superior de Huejutla, Email: yarelii.zurita@gmail.com, adanellyzh@gmail.com, arturoalarcon23727@gmail.com, leo961022@gmail.com

${ }^{\mathrm{c}}$ Universidad Autónoma del Estado de Hidalgo, Escuela Superior de Huejutla, Email: raul_palacios@uaeh.edu.mx 
red, el cliente es el que inicia la comunicación, mientras que el programa que espera ser contactado es el servidor. Cualquier programa puede actuar como servidor para un servicio y como cliente para otro [8].

\section{Servidor}

un Servidor es el equipo que aloja una gran variedad y un alto índice de datos, además de brindar una mayor seguridad y confianza para que los archivos allí alojados no se perderán o filtrarán [9]

En la presente práctica, basado en la guía de instalación [10] y configuración de NFS, se proporciona e procedimiento de instalación de NFS en una red local de 5 nodos en una distribución de Linux.

\section{Requerimientos}

Para llevar a cabo la práctica se usaron equipos con las siguientes características:

- Procesador: Intel(R) Core (TM) i5-6500 CPU @ $3.20 \mathrm{GHz} 3.20 \mathrm{GHz}$

- RAM: $4.00 \mathrm{~GB}$

- Disco Duro 1 TB

- Sistema Operativo CentOS 7

- Switch Cisco SG220-50 50-Port Gigabit Smart Plus

\section{Desarrollo}

En primer lugar, es necesario definir las direcciones IP que se van a utilizar tanto para el servidor y los clientes. En nuestro caso se representan de la siguiente manera (Tabla 1):

\begin{tabular}{|l|l|l|l|}
\hline Nodo & IP & Máscara de red & $\begin{array}{l}\text { Puerta de } \\
\text { enlace }\end{array}$ \\
\hline servidor & 10.13 .21 .2 & 255.255 .255 .0 & 10.13 .21 .1 \\
\hline nodo1 & 10.13 .21 .3 & 255.255 .255 .0 & 10.13 .21 .1 \\
\hline nodo2 & 10.13 .21 .4 & 255.255 .255 .0 & 10.13 .21 .1 \\
\hline nodo3 & 10.13 .21 .5 & 255.255 .255 .0 & 10.13 .21 .1 \\
\hline nodo4 & 10.13 .21 .6 & 255.255 .255 .0 & 10.13 .21 .1 \\
\hline
\end{tabular}

Tabla 1. Direcciones IP de los nodos de la configuración.

\section{Instalación y configuración del servidor NFS}

1.- Primeramente, se instalan los paquetes desde la consola, los comandos se ejecutan con el usuario root. En CentOS se utiliza el comando yum para la instalación. como se muestra en la siguiente ilustración.

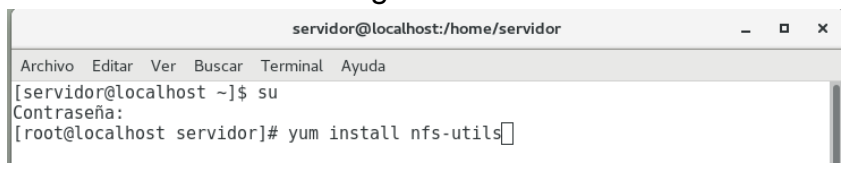

2.- A continuación, se crea el directorio a compartir por el servidor NFS, tal como se muestra en la siguiente ilustración.

\begin{tabular}{l}
\multicolumn{1}{c|}{ servidor@localhost:/home/servidor } \\
Archivo Editar Ver Buscar Terminal Ayuda \\
[servidor@localhost $\sim$ ] $\mathrm{su}$ \\
Contraseña: \\
[root@localhost servidor]\# mkdir /var/nfsshare]
\end{tabular}

3.- Se cambian los permisos de la carpeta de la siguiente manera:

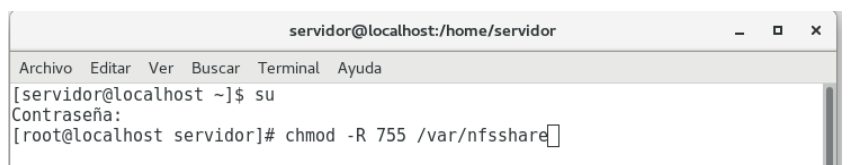

La ilustración anterior muestra el comando utilizado para cambiar los permisos.

servidor@localhost:/home/servidor

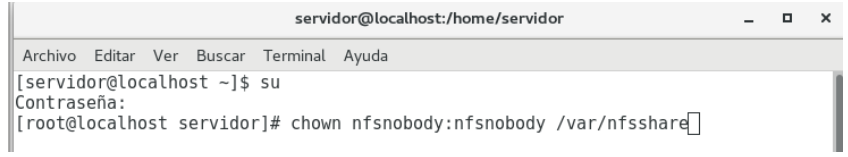

4.- Como siguiente paso, se inician los servicios y se permite que inicien en el momento del arranque del sistema.

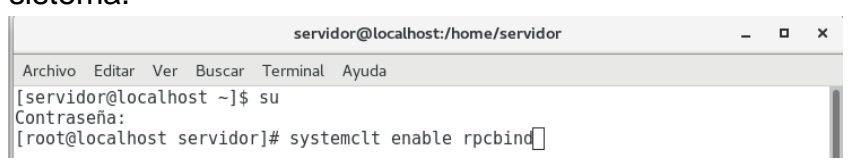

La ilustración anterior muestra cómo se inicia el servicio.
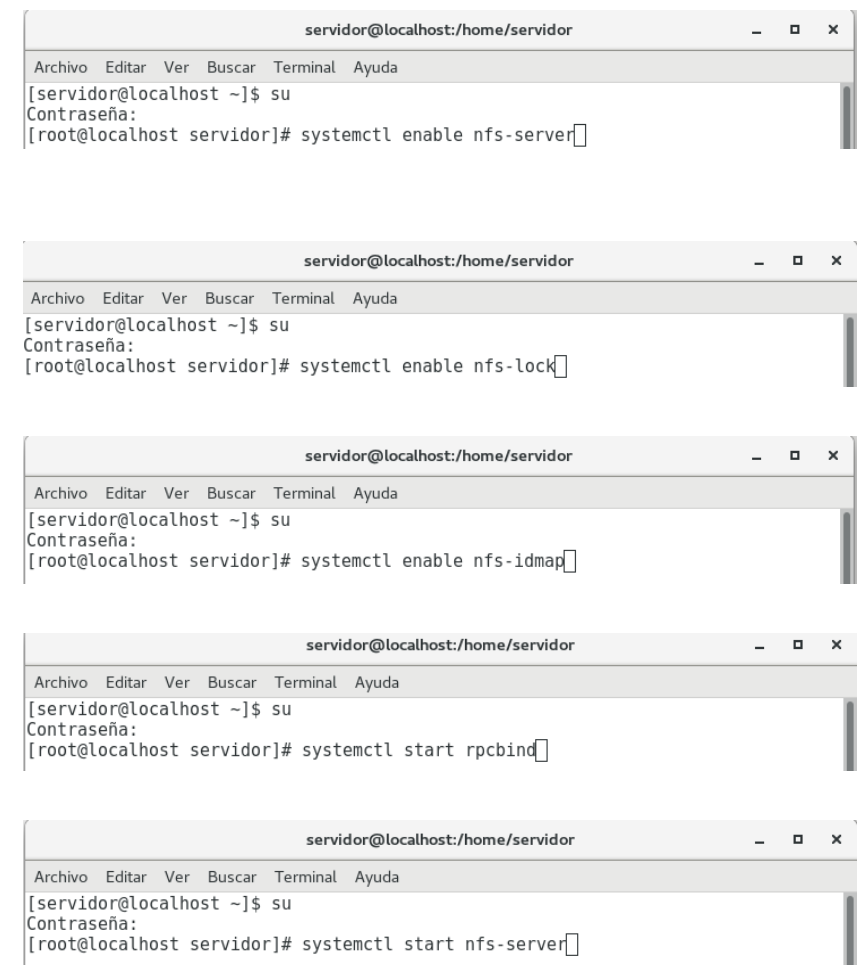

5.- Se modifica el archivo/etc/exports, aquí es donde se colocan todos los directorios necesarios para que estén disponibles con NFS, este archivo es importante ya que aquí se definen algunos permisos y protocolos a usar. 


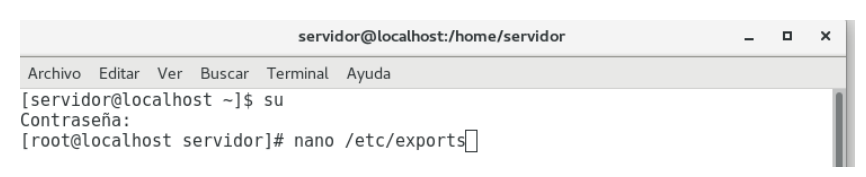

6.- En este archivo se crean dos puntos para compartir los cuales serán /home y /var/nfsshare. Editamos el archivo exports de la siguiente manera.

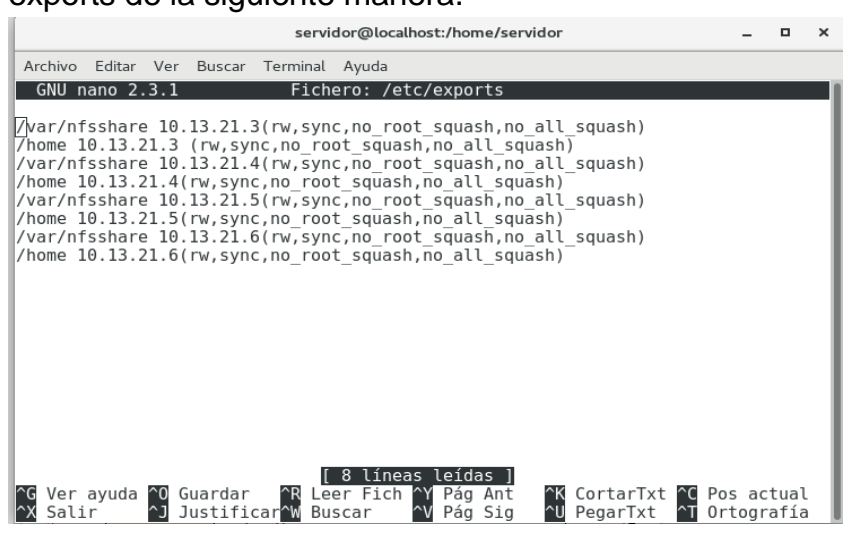

Donde las direcciones IP que contiene este archivo identifican los nodos cliente.

\begin{tabular}{|l|l|}
\hline Opción & Descripción \\
\hline rw & $\begin{array}{l}\text { Permitir las solicitudes de lectura y escritura en volumen } \\
\text { NFS. }\end{array}$ \\
\hline sync & $\begin{array}{l}\text { Responsa a las solicitudes solo después de que los } \\
\text { cambios se hayan confirmado en el almacenamiento } \\
\text { estable. }\end{array}$ \\
\hline no_root_squash & $\begin{array}{l}\text { Si se selecciona no_root_squash, el root en la máquina } \\
\text { cliente tendrá el mismo nivel de acceso a los archivos } \\
\text { del sistema como root en el servidor. }\end{array}$ \\
\hline no_all_squash & $\begin{array}{l}\text { Esta opción es principalmente útil para clientes sin } \\
\text { disco. }\end{array}$ \\
\hline
\end{tabular}

Tabla 2. Opciones de exportación en NFS.

7.- Las opciones de exportación que estamos otorgando a los clientes y que definimos como necesarias para nuestro servidor NFS se mencionan en la Tabla 2.

8.- Una vez realizada la configuración, es necesario que los cambios realizados tomen efecto. Por lo cual, es necesario reiniciar el servicio.

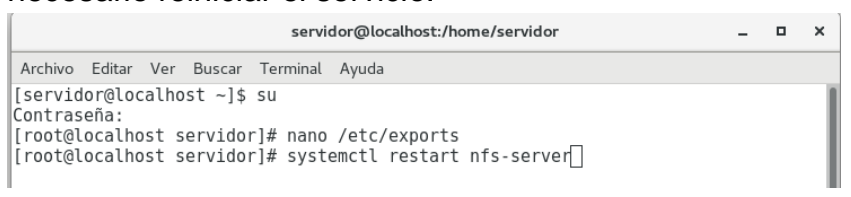

9.- Es necesario agregar reglas de firewall para que no bloquee el acceso desde el cliente.

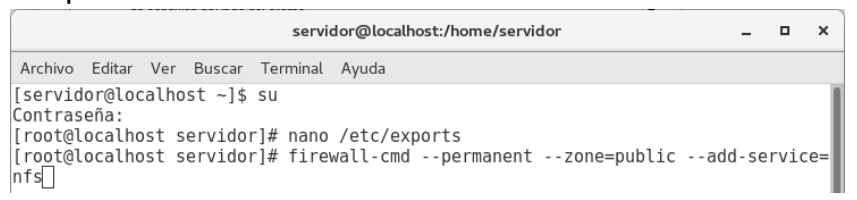

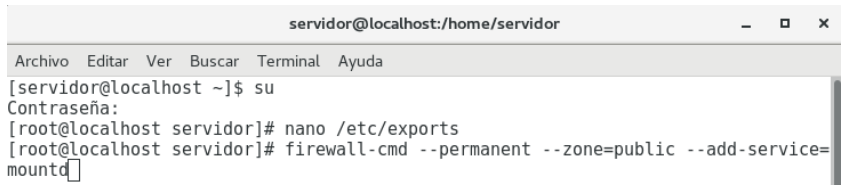

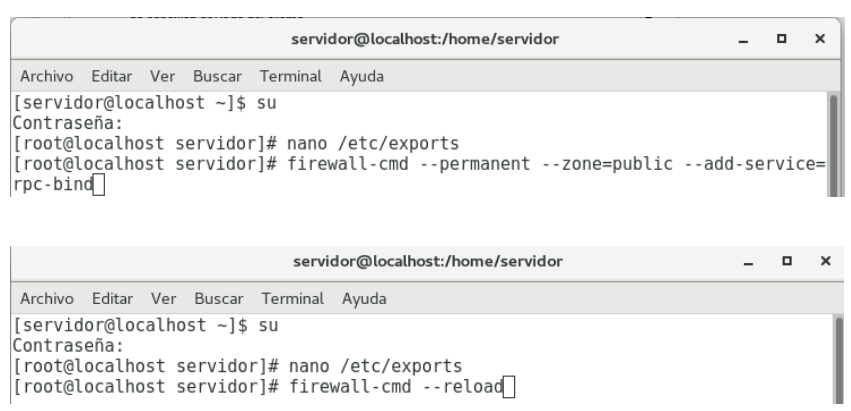

La configuración de firewall es necesario ya que, si no se hace, dará un error para el tiempo de espera de conexión del lado del cliente.

\section{Instalación y configuración del cliente NFS}

10.- En el cliente desde donde queremos acceder al servidor NFS es necesario instalar las utilidades de NFS.

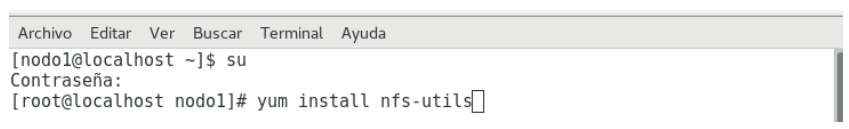

11.- A continuación, se crean los puntos de montaje del directorio NFS:

\begin{tabular}{|c|c|c|c|}
\hline & nodo1@localhost:/home/nodo1 & $-\quad \square$ & $x$ \\
\hline Archivo Editar Ver Buscar & Terminal Ayuda & & \\
\hline \multicolumn{4}{|c|}{$\begin{array}{l}\text { [nodol@localhost ]\$ su } \\
\text { Contraseña: } \\
\text { [root@localhost nodol]\# mkdir -p /mnt/nfs/home] }\end{array}$} \\
\hline & nodo1@localhost:/home/nodo1 & - & $x$ \\
\hline Archivo Editar Ver Buscar & Terminal Ayuda & & \\
\hline
\end{tabular}

12.- A continuación, montaremos el directorio /home compartido de NFS en el nodo cliente como usuario root.

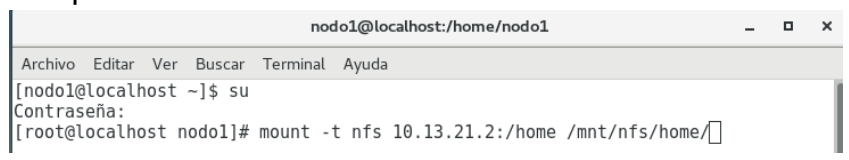

13.- Ahora solo falta montar el directorio /var/nfsshare.

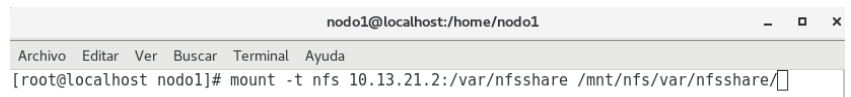

14.- Se verifica la conexión con el servidor NFS con el siguiente comando:

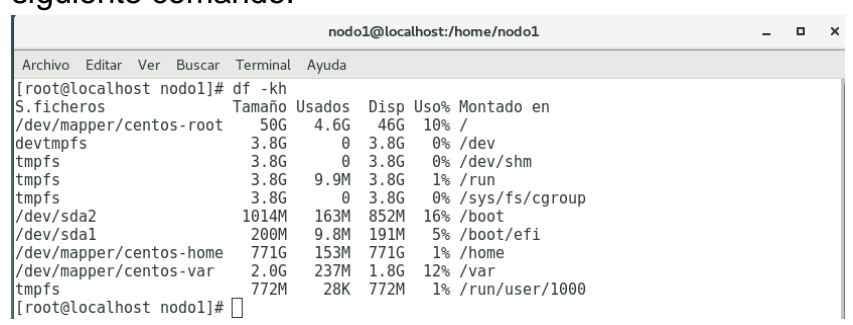


15.- Ahora verifica que se tienen los permisos de lectura/escritura en la ruta compartida. Para ello colocamos en la consola el siguiente comando:

Archivo Editar Ver Buscar Terminal Ayuda

[root@localhost nodo1]\# touch /mnt/nfs/var/nfsshare/test_nfs[

Ahora se tienen configurados los nodos cliente NFS. Cabe resaltar que, esta configuración se realizó en cada uno de los nodos cliente.

\section{Montaje del NFS permanente}

16.- Para no cargar NFS cada vez que reinicie el sistema en los nodos, es necesario modificar el archivo /etc/fstab de los nodos cliente para que se autocargue.

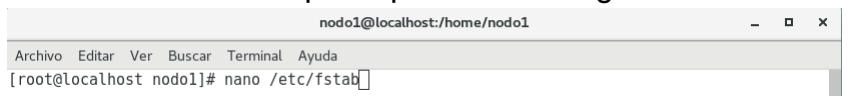

17.- Se agrega lo siguiente en cada nodo cliente:

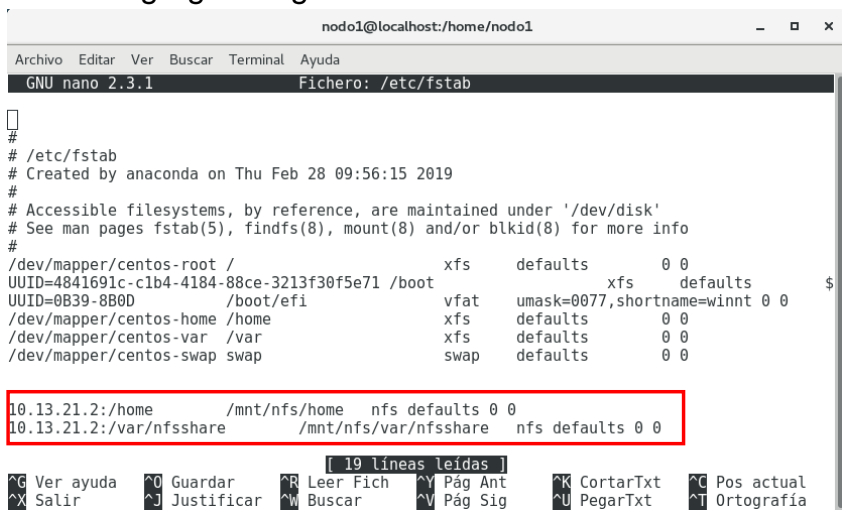

Donde 10.13.21.2 es la dirección IP del servidor NFS.

Con esto se hará un montaje permanente de NFS-share. De esta manera se configura tanto el cliente como el servidor sobre CentOS 7.

\section{Arranque automático de NFS al iniciar el sistema}

18.- De modo predeterminado el servicio NFS estará inactivo. Para activar este servicio en los niveles de ejecución 3, 4 y 5, es decir los niveles recomendados, ejecutamos lo siguiente:

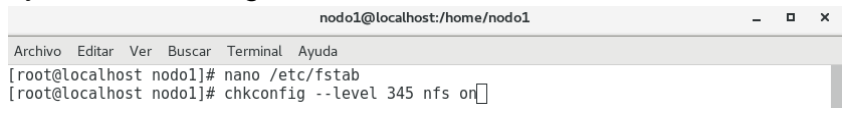

Con esto ya no será necesario iniciar el servicio cada que se reinicie los nodos cliente.

\section{Pruebas}

19.- Para verificar que el servicio NFS esté funcionando correctamente, solo debemos de crear un archivo desde el servidor y observar que en los nodos cliente se pueda leer y editar.

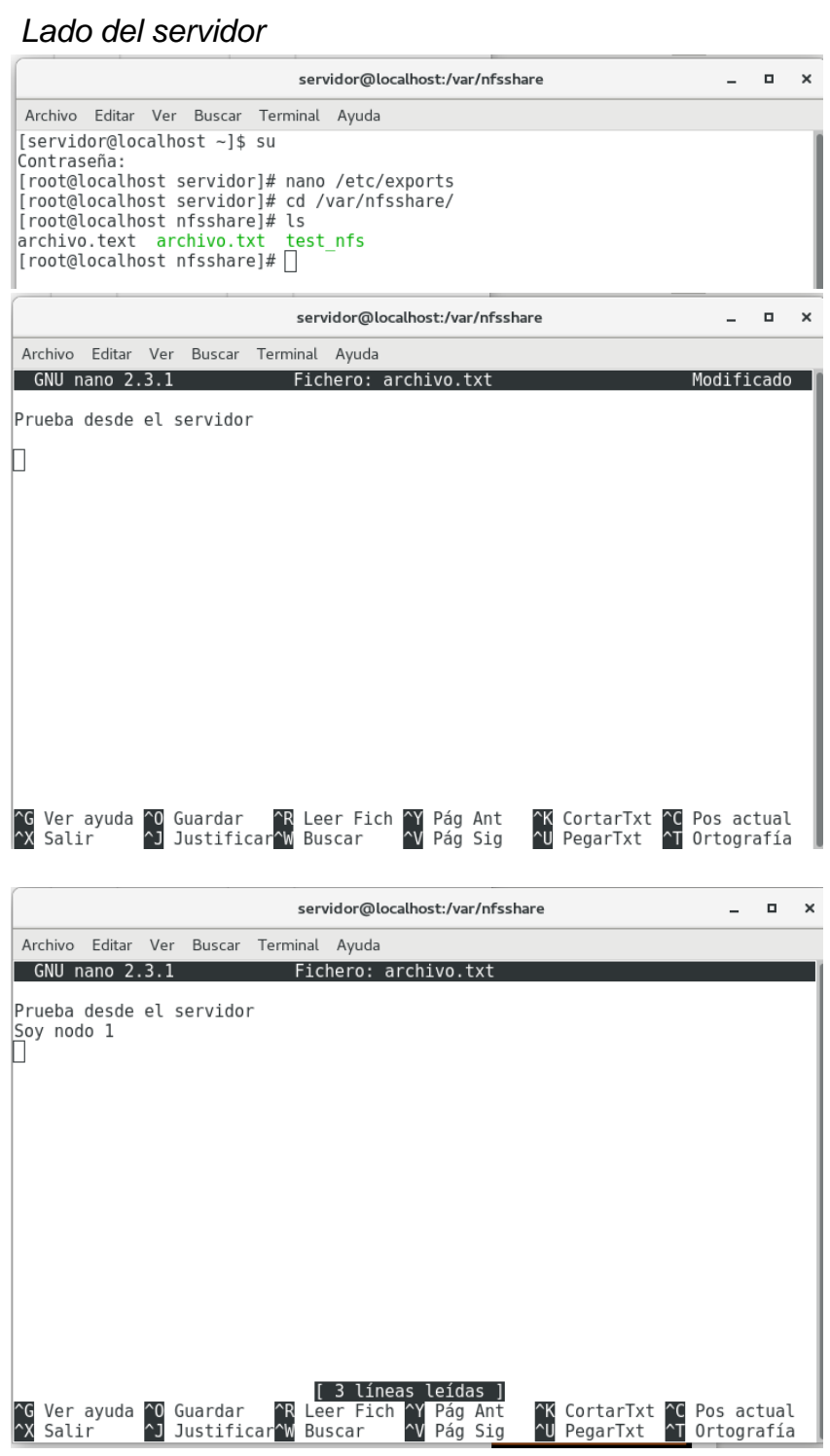

Lado del cliente

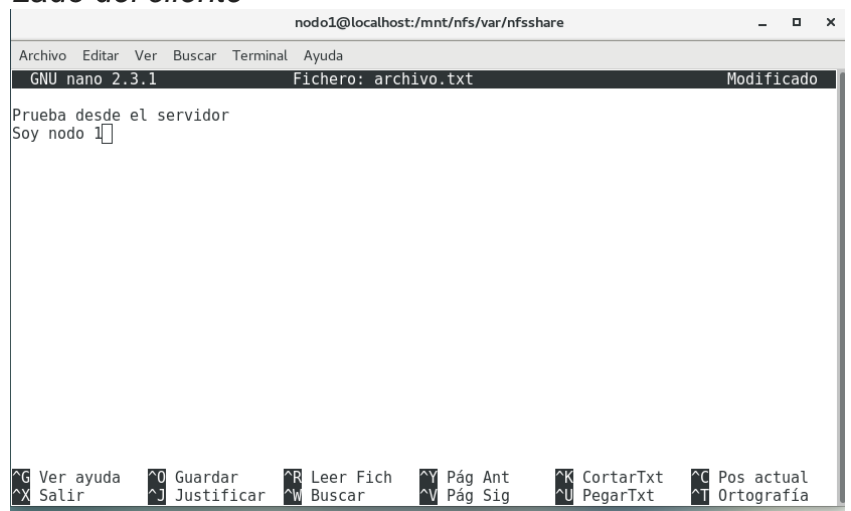




\section{Conclusión}

Al término de la práctica, se aprendió a crear un servidor NFS (Network File System), el cual es utilizado para sistemas de archivos distribuidos en un entorno de red de computadoras de área local, el servidor NFS permite a los usuarios acceder a los archivos y directorios ubicados en sistemas remotos y tratar dichos archivos y directorios como si fueran locales.

El usuario accede de manera remota a través de los directorios y esta puede acceder a los archivos, si el nodo cliente tiene permisos desde el nodo servidor, podrá acceder de manera remota y editar dichos archivos que se encuentren dentro de la carpeta compartida desde el servidor.

En el desarrollo de esta práctica, no existieron complicaciones graves, como recomendación se debe tener cuidado al momento de configurar el servidor ya que los comandos y acciones pueden variar un poco de la configuración del cliente y podrían generar algunos errores para la comunicación entre el servidor y los clientes, debido a una mala configuración.

\section{Referencias}

[1] R. Herrold, "Acerca de CentOS." [Online]. Available: https://www.centos.org/about/. [Accessed: 02-May-2019].

[2] Margaret Rouse, “¿Qué es Sistema de archivos de red, NFS? - Definición en Whatls.com." [Online]. Available: https://searchdatacenter.techtarget.com/es/def inicion/Sistema-de-archivos-de-red-NFS.

[Accessed: 29-Mar-2019].

[3] J. Raul and N. Fonseca, "INSTITUTO TECNOLÓGICO Y DE ESTUDIOS SUPERIORES DE MONTERREY CAMPUS ESTADO DE MEXICO FORTALECIMIENTO DE NFS Y COMPARACION CON OTROS SISTEMAS DE ARCHIVOS SEGUROS. TESIS QUE PRESENTA."

[4] "NFS: Sistema de archivos de red Observatorio Tecnológico." [Online]. Available: http://recursostic.educacion.es/observatorio/w eb/gl/software/software-general/733-nfssistema-de-archivos-de-red. [Accessed: 28Mar-2019].

[5] R. M. G. Labrador and M. De, "4127 ADMINISTRACIÓN DE SERVIDORES LINUX (UBUNTU/FEDORA/CENTOS)."

[6] M. Ramos Montes, "Servidor de Internet de alta disponibilidad con equilibrado de carga," Sep. 2018.
[7] N. Nishimura, 西村則康, J. Paz Campaña, and R. Romero Navarrete, Tomobataraki dakara dekiru chugaku juken hisshoho. Asashuppan, 2018.

[8] S. Master, "Definición de Cliente - Significado y definición de Cliente." [Online]. Available: https://sistemas.com/cliente.php. [Accessed: 28-Mar-2019].

[9] S. Master, "Definición de Red Cliente / Servidor - Significado y definición de Red Cliente / Servidor." [Online]. Available: https://sistemas.com/red-cliente-servidor.php. [Accessed: 28-Mar-2019].

[10] "Instalación de servidor y cliente NFS en CentOS 7." [Online]. Available:

https://www.howtoforge.com/nfs-server-andclient-on-centos-7. [Accessed: 28-Mar-2019]. 